\title{
BMJ Open The effects of sodium-glucose co-transporter 2 inhibitors in patients with type 2 diabetes: protocol for a systematic review with meta-analysis of randomised trials
}

\author{
Heidi Storgaard, ${ }^{1}$ Lise Lotte Gluud, ${ }^{2}$ Mikkel Christensen, ${ }^{1,3}$ Filip Krag Knop,,${ }^{1,4}$ \\ Tina Vilsbøll ${ }^{1}$
}

To cite: Storgaard $\mathrm{H}$, Gluud LL, Christensen M, et al. The effects of sodiumglucose co-transporter 2 inhibitors in patients with type 2 diabetes: protocol for a systematic review with meta-analysis of randomised trials. BMJ Open 2014;4 e005378. doi:10.1136/ bmjopen-2014-005378

- Prepublication history for this paper is available online. To view these files please visit the journal online (http://dx.doi.org/10.1136/ bmjopen-2014-005378)

Received 2 April 2014 Revised 11 June 2014 Accepted 13 June 2014

CrossMark

For numbered affiliations see end of article.

Correspondence to Dr Heidi Storgaard; heidi.storgaard@regionh.dk

\section{ABSTRACT}

Introduction: Sodium-glucose co-transporter 2 inhibitors (SGLT-2i) increase urinary glucose excretion through a reduced renal glucose reabsorption. We plan to perform a systematic review of SGLT-2i for treatment of type 2 diabetes.

Methods and analysis: A systematic review with meta-analyses of randomised clinical trials on SGLT-2i versus placebo, other oral glucose lowering drugs or insulin for patients with type 2 diabetes will be performed. The primary end point will be the glycated haemoglobin. Secondary end points will include changes in body weight, body mass index, fasting plasma glucose, plasma cholesterol, kidney and liver blood tests, blood pressure and adverse events. Electronic (the Cochrane Library, MEDLINE, EMBASE and the Science Citation Index) and manual searches will be performed. Meta-analyses will be performed and the results presented as mean differences for continuous outcomes and risk differences for dichotomous outcomes, both with $95 \% \mathrm{Cls}$. Subgroup, sensitivity, regression and sequential analyses will be performed to evaluate intertrial heterogeneity, bias and the robustness of results due to cumulative testing.

Ethics and dissemination: The study will contribute to the knowledge regarding the beneficial and harmful effects of SGLT-2i in patients with type 2 diabetes. We plan to publish the study irrespective of the results.

Results: The study will be disseminated by peerreview publication and conference presentation.

Trial registration number: PROSPERO CRD42014008960

\section{INTRODUCTION}

Type 2 diabetes is a metabolic disease associated with obesity, dyslipidaemia and hypertension. Patients with type 2 diabetes are characterised by defective insulin secretion, insulin resistance, inappropriate glucagon secretion and an impaired incretin

\section{Strengths and limitations of this study}

- We have the knowledge and experience on how to conduct a systematic review and meta-analysis.

- We limit our analyses to only include trials on the relevant daily doses of SGLT-2i to give the evidence-based clinician a more useful answer.

- A possible limitation might be the access to data from the randomised clinical trials we plan to include in the study.

effect resulting in fasting and postprandial hyperglycaemia. ${ }^{1}$ Hyperglycaemia with elevated levels of glycated haemoglobin (HbA1c) predicts microvascular and macrovascular complications. ${ }^{2}$ Although improved metabolic control is associated with reduced morbidity and mortality, ${ }^{3}$ recent studies show that intensive glucose lowering treatments may harm some patients. ${ }^{4-7}$ As a consequence, the American Diabetes Association (ADA) and the European Association for the Study of Diabetes (EASD) recommend individualisation of the treatment. ${ }^{8}$ Drugs with complementary mechanisms of action are recommended with metformin as a first-line therapy. As $\beta$-cell function declines, a number of patients fail to achieve their glycaemic target and maintenance of glucose control often necessitates several add-on therapies. $^{8}$ Current oral medications endorsed by ADA and EASD treatment algorithms for treating patients with type 2 diabetes, that is, metformin, sulfonylureas, dipeptidyl peptidase 4 inhibitors and thiazolidinediones, act by increasing insulin secretion or sensitising tissues to insulin action. Treatment strategies with insulin-independent pathways could therefore be advantageous. 
Sodium-glucose co-transporter 2 inhibitors (SGLT-2i) represent a new class of drugs that inhibit glucose reabsorption in the proximal tubules of the kidneys. As a result, urinary glucose excretion is increased, which in turn reduces the amount of circulating glucose and improves glycaemic control. The effect is not associated with insulin secretion or action. ${ }^{9}$

In clinical trials, SGLT-2i (in monotherapy or combined with metformin, sulfonylureas, pioglitazone or insulin) seems to improve glycaemic control in type 2 diabetes. ${ }^{10-14}$ In 2013 and 2014, two STGL-2i, canagliflozin and dapagliflozin, were approved by the US Food and Drug Administration (FDA) ${ }^{15}{ }^{16}$ and the European Medicine Agency (EMA) for the treatment of patients with type 2 diabetes. ${ }^{17} 18$ None of the individual clinical trials on SGLT-2i provide definite conclusions regarding efficacy and safety, and so far the current guidelines for the management of type 2 diabetes do not include SGLT-2i. ${ }^{8}$ In order to provide robust evidence for the efficacy and safety of SGLT-2i, we plan to perform a systematic review with meta-analyses of randomised controlled trials (RCTs). Previous systematic reviews on SGLT-2i ${ }^{19-24}$ used a pragmatic approach and included trials irrespective of the dosing or duration of follow-up. We restricted our analyses to 'clinically relevant' trials, that is, trials assessing doses and interventions that we use in clinical practice. We therefore limit our analyses to include trials on the recommended daily dose, clinical relevant compounds and with sufficient follow-up to assess the clinical effects. This approach means that smaller trials, such as dose finding trials, will not be included. We believe that this approach will give the evidence-based clinician a clearer and more useful answer. Doses that are not clinically relevant may underestimate or overestimate the beneficial and potentially harmful effects of SGLT-2i. Therefore our data may provide a more accurate answer that may be used in clinical practice.

\section{OBJECTIVES}

The primary objective of this systematic review is to evaluate the effects of SGLT-2i that are approved (dapagliflozin and canagliflozin) or are in late clinical development (empagliflozin) in Europe and the USA. To increase external validity, we plan to evaluate doses that are currently recommended by FDA and/or EMA EM $^{172} 26$ as a maximum daily dose and therefore only include trials with these daily doses (canagliflozin $300 \mathrm{mg}$, dapagliflozin $10 \mathrm{mg}$ and empagliflozin $25 \mathrm{mg}$ ). Our primary objective will be to assess the impact on glycaemic control (HbA1c).

\section{METHODS}

The reporting of the review will follow the preferred reporting items for systematic reviews and meta-analysis (PRISMA) statement. ${ }^{27}$
Criteria for considering studies for this review

Studies

The review will include RCTs irrespective of blinding and language.

\section{Participants}

Adult patients (at least 18 years of age) of both genders with type 2 diabetes will be included.

\section{Duration}

Since red blood cells survive for 8-12 weeks, the trials should last for at least 12 weeks to evaluate the effect of SGLT-2i on HbA1c.

\section{Interventions}

The intervention comparisons will constitute SGLT-2i (dapagliflozin, canagliflozin and empagliflozin) versus placebo, other oral antidiabetic drugs or insulin. Co-interventions with other antidiabetic agents will be allowed if administered to the intervention and control groups.

\section{Types of outcome measures}

The following outcome measures will be assessed

Primary outcome measure

- HbAlc

\section{Secondary outcome measures}

- Body weight and body mass index (BMI)

- Fasting plasma glucose

- Lipid profile (low-density lipoprotein (LDL) cholesterol, high-density lipoprotein (HDL) cholesterol and triglyceride)

- Systolic and diastolic blood pressure

- Liver and kidney blood tests (creatinine and uric acid)

- Urinary albumin

- Adverse events (any adverse events, urinary tract infections, genital tract infections, hypoglycaemia, hypotension, total withdrawals)

\section{Search methods for identification of studies}

All authors will participate in the identification and selection of trials. Excluded trials will be listed with the reason for exclusion. Authors will extract data in an independent manner. Eligible trials will be identified through electronic and manual searches. Electronic searches will be performed in MEDLINE ((Sodiumglucose (All Fields) AND co-transporter (All Fields)) OR ("2-(3-(4-ethoxybenzyl)-4-chlorophenyl)-6-hydroxymethyltetrahydro-2H-pyran-3,4,5-triol" (Supplementary Concept) OR "2-(3-(4-ethoxybenzyl)-4-chlorophenyl)-6hydroxymethyltetrahydro-2H-pyran-3,4,5-triol" (All Fields) OR “dapagliflozin" (All Fields)) OR ("canagliflozin" (Supplementary Concept) OR "canagliflozin" (All Fields)) OR ("empagliflozin" (Supplementary Concept) OR "empagliflozin” (All Fields)) OR Remogliflozin (All Fields) OR 
("sergliflozin" (Supplementary Concept) OR "sergliflozin" (All Fields)) OR ("6-((4-ethylphenyl)methyl)-3', $4^{\prime}, 5^{\prime}, 6^{\prime}$-tetrahydro-6'-(hydroxymethyl ) spiro (isobenzofuran-1 (3H), 2'(2H) pyran)- $3^{\prime}, 4^{\prime}, 5^{\prime}$-triol” (Supplementary Concept) OR "6-((4-ethylphenyl) methyl)-3', 4',5',6'-tetrahydro$6^{\prime}$-(hydroxymethyl) spiro (isobenzofuran-1 $(3 \mathrm{H}), 2^{\prime}-(2 \mathrm{H})$ pyran)-3', $4^{\prime}, 5^{\prime}$-triol" (All Fields) OR "tofogliflozin" (All Fields))), Cochrane Library, Embase and Web of Science. Additional manual searches will be performed in reference lists of relevant papers, correspondence with experts, the pharmaceutical companies producing SGLT-2i and the WHO Trial Search Database. ${ }^{28}$

\section{Data collection and analysis}

Two authors (HS and MC) will independently extract data and resolve disagreements through discussion before analysis. In the case of unresolved matters, a third party (TV, FKK or LLG) will be involved. If necessary data are not included in the published trial reports, authors of included trials will be contacted for additional information.

\section{Selection of studies}

Trials identified through searches will be listed and selected for inclusion according to the aforementioned criteria.

\section{Data extraction}

Extraction forms developed for the study will be used and the following data will be extracted: trial characteristics (number of clinical sites, country of origin and funding), intervention characteristics (type, dose and duration of interventions applied), patient characteristics (inclusion criteria, background treatment, mean age, proportion of men, duration of type 2 diabetes, body weight, BMI, baseline systolic and diastolic blood pressure, urinary albumin baseline HbAlc, baseline blood tests, fasting plasma glucose, LDL-cholesterol, HDL-cholesterol and triglyceride alanine amino transferase, alkaline phosphatase, creatinine and urate).

\section{Assessment of risk of bias in included studies}

The bias risk assessment will follow the recommendations described in the Cochrane Handbook for Systematic Reviews of Interventions and includes:

- Randomisation (selection bias): randomisation methods will be extracted as the primary measure of bias control. ${ }^{29}$ Methodological quality in randomisation methods will be based on allocation sequence generation (adequate if based on a table of random numbers, computer-generated random numbers or similar) and allocation concealment (adequate if randomisation was performed through serially numbered opaque sealed envelopes, a central independent unit, identically appearing coded drug containers or similar).
- Blinding (performance and detection bias): we will extract data on whether single or double blinding was performed, the method of blinding (eg, use of placebo) and the persons who were blinded with regard to the interventions assessed (eg, healthcare providers or patients).

- Incomplete outcome data (attrition bias): the extent to which all patients lost to follow-up are accounted for.

- Outcome reporting (reporting bias): the extent to which clinically relevant outcome measures are reported and differences between trial protocols and subsequent reports will be evaluated as a marker of reporting bias.

- Other bias: any other apparent biases will be evaluated.

\section{Statistical analyses}

Analyses will be performed in RevMan ${ }^{30}$ and Stata V.13 (STATA Corp, College Station, Texas, USA). They will be based on individual patient data when available or on published data. $\mathrm{I}^{2}$ will be used as a measure of heterogeneity. $\mathrm{I}^{2}$ values below $30 \%$ will be defined as unimportant, $30-50 \%$ as moderate, $50-75 \%$ as substantial and $>75 \%$ as considerable heterogeneity. Irrespective of the statistical heterogeneity, the fixed effects and random effects models will be used to test the robustness of the results. We will only report the results of the random effects meta-analyses if the results differ from those of the fixed effects models. Publication bias and other small study effects will be evaluated based on regression analysis (Egger's or Harbord's test).

We plan to perform subgroup and meta-regression analyses based on treatment combinations as well as baseline patient characteristics. Differences between subgroups will be explored using tests for subgroup differences expressed as $p$ values. The subgroup analyses will evaluate the influence of the type of data (individual patient data or published data), the control groups (stratified by the type of intervention allocated to the control group) and collateral interventions (interventions administered to both allocation groups). We will also perform meta-regression analyses to evaluate the potential influence of glycaemic control at baseline, duration of diabetes and baseline body weight. In sensitivity analyses, we will evaluate the intervention effect in: 1) patients who are of normal weight (defined as a maximum BMI of $25 \mathrm{~kg} / \mathrm{m}^{2}$ ), 2) trials published as full paper articles and 3) trials with a low risk of bias.

\section{Measures of treatment effect}

Dichotomous data will be analysed using risk differences and continuous data using mean differences, both with $95 \%$ CIs.

\section{Unit of analyses issues}

For trials presenting data from more than one treatment period (eg, 26 and 52 weeks), data from the longest 
treatment period will be used. On the basis of the primary outcome measure, we will only use data from the first period of cross-over trials.

\section{Dealing with missing data}

Intention-to-treat analyses including all patients randomised will be performed. For patients with missing data, we will perform sensitivity analyses with simple imputation (counting patients as failures or successes).

\section{ETHICS AND DISSEMINATION}

The study will evaluate the clinical effect of SGLT-2i in patients with type 2 diabetes based on the available published and unpublished clinical trial data and thereby potentially improve the clinical knowledge on and management of type 2 diabetes.

\section{Author affiliations}

${ }^{1}$ Center for Diabetes Research, Department of Medicine, Gentofte Hospital, University of Copenhagen, Hellerup, Denmark

${ }^{2}$ Gastrounit, Hvidovre Hospital, University of Copenhagen, Hvidovre, Denmark ${ }^{3}$ Department of Clinical Pharmacology, Bispebjerg Hospital, University of Copenhagen, Copenhagen, Denmark

${ }^{4}$ Department of Biomedical Sciences, Faculty of Health and Medical Sciences, University of Copenhagen, Copenhagen, Denmark

Contributors HS, LLG, MC, FKK and TV participated in the conception and design of this protocol including search strategy development. LLG provided statistical advice for the design. HS prepared the draft and all authors reviewed the manuscript and approved the final version.

Funding TV and FKK are supported by unrestricted grants from the Novo Nordisk Foundation. The research did not otherwise receive specific grant from any funding agency in the public, commercial or not-for-profit sectors. No sponsor was involved in study design, and no sponsor will have authority in collection, management, analysis and interpretation of data. Writing of the report and the decision to submit the results for publication is strictly made by the authors.

Competing interests None.

Patient consent Obtained.

Provenance and peer review Not commissioned; externally peer reviewed.

Open Access This is an Open Access article distributed in accordance with the Creative Commons Attribution Non Commercial (CC BY-NC 4.0) license, which permits others to distribute, remix, adapt, build upon this work noncommercially, and license their derivative works on different terms, provided the original work is properly cited and the use is non-commercial. See: http:// creativecommons.org/licenses/by-nc/4.0/

\section{REFERENCES}

1. DeFronzo RA. From the triumvirate to the ominous octet: a new paradigm for the treatment of type 2 diabetes mellitus. Diabetes 2009;58:773-95.

2. Gaede $\mathrm{P}$, Vedel $\mathrm{P}$, Larsen $\mathrm{N}$, et al. Multifactorial intervention and cardiovascular disease in patients with type 2 diabetes. $N$ Engl $J$ Med 2003;348:383-93.

3. Stratton IM, Adler AI, Neil HA, et al. Association of glycaemia with macrovascular and microvascular complications of type 2 diabetes (UKPDS 35): prospective observational study. BMJ 2000;321:405-12.

4. Gerstein HC, Miller ME, Byington RP, et al.; Action to Control Cardiovascular Risk in Diabetes Study Group. Effects of intensive glucose lowering in type 2 diabetes. $N$ Engl J Med 2008:358:2545-59.

5. Patel A, MacMahon S, Chalmers J, et al.; ADVANCE Collaborative Group. Intensive blood glucose control and vascular outcomes in patients with type 2 diabetes. N Engl J Med 2008;358:2560-72.
6. Duckworth W, Abraira C, Moritz T, et al. Glucose control and vascular complications in veterans with type 2 diabetes. $N$ Engl $J$ Med 2009;360:129-39.

7. Holman RR, Paul SK, Bethel MA, et al. 10-year follow-up of intensive glucose control in type 2 diabetes. N Engl J Med 2008;359:1577-89.

8. Inzucchi SE, Bergenstal RM, Buse JB, et al. Management of hyperglycaemia in type 2 diabetes: a patient-centered approach. Position statement of the American Diabetes Association (ADA) and the European Association for the Study of Diabetes (EASD). Diabetologia 2012;55:1577-96.

9. Ferrannini E, Solini A. SGLT2 inhibition in diabetes mellitus: rationale and clinical prospects. Nat Rev Endocrinol 2012;8:495-502.

10. Ferrannini E, Ramos SJ, Salsali A, et al. Dapagliflozin monotherapy in type 2 diabetic patients with inadequate glycemic control by diet and exercise: a randomized, double-blind, placebo-controlled, phase 3 trial. Diabetes Care 2010;33:2217-24.

11. Rosenstock J, Aggarwal N, Polidori D, et al. Dose-ranging effects of canagliflozin, a sodium-glucose cotransporter 2 inhibitor, as add-on to metformin in subjects with type 2 diabetes. Diabetes Care 2012;35:1232-8.

12. Häring $\mathrm{H}-\mathrm{U}$, Merker L, Seewaldt-Becker E, et al. Empagliflozin as add-on to metformin plus sulfonylurea in patients with type 2 diabetes: a 24-week, randomized, double-blind, placebo-controlled trial. Diabetes Care 2013;36:3396-404.

13. Kovacs CS, Seshiah V, Swallow R, et al. Empagliflozin improves glycaemic and weight control as add-on therapy to pioglitazone or pioglitazone plus metformin in patients with type 2 diabetes: a 24-week, randomized, placebo-controlled trial. Diabetes Obes Metab 2014;16:148-57.

14. Wilding JPH, Woo V, Rohwedder K, et al. Dapagliflozin in patients with type 2 diabetes receiving high doses of insulin: efficacy and safety over 2 years. Diabetes Obes Metab 2014;16:124-36.

15. EMDAC FDA Backgrounder-UCM378076.pdf. http://www.fda.gov/ downloads/AdvisoryCommittees/CommitteesMeetingMaterials/ Drugs/EndocrinologicandMetabolicDrugsAdvisoryCommittee/ UCM378076.pdf

16. UCM334550.pdf. http://www.fda.gov/downloads/AdvisoryCommittees/ CommitteesMeetingMaterials/Drugs/EndocrinologicandMetabolicDrugs AdvisoryCommittee/UCM334550.pdf

17. Forxiga, INN-dapagliflozin-WC500136024.pdf. http://www.ema. europa.eu/docs/en_GB/document_library/EPAR_-_Public_assessment report/human/002322/WC500136024.pdf

18. Invokana_-_CHMP_AR_final-WC500156457.pdf. http://www.ema. europa.eu/docs/en GB/document library/EPAR - Public assessment_report/human/002649/WC500156457.pdf

19. Vasilakou D, Karagiannis T, Athanasiadou E, et al. Sodium-glucose cotransporter 2 inhibitors for type 2 diabetes: a systematic review and meta-analysis. Ann Intern Med 2013;159:262-74.

20. Berhan A, Barker A. Sodium glucose co-transport 2 inhibitors in the treatment of type 2 diabetes mellitus: a meta-analysis of randomized double-blind controlled trials. BMC Endocr Disord 2013;13:58.

21. Clar C, Gill JA, Court R, et al. Systematic review of SGLT2 receptor inhibitors in dual or triple therapy in type 2 diabetes. BMJ Open 2012;2,pii:001007.

22. Goring S, Hawkins N, Wygant G. Dapagliflozin compared with other oral anti-diabetes treatments when added to metformin monotherapy: a systematic review and network meta-analysis. Diabetes Obes Metab 2014;16:433-42.

23. Liakos A, Karagiannis T, Athanasiadou E, et al. Efficacy and safety of empagliflozin for type 2 diabetes: a systematic review and metaanalysis. Diabetes Obes Metab Published Online First: 26 April 2014, doi:10.1111/dom.12307

24. Musso G, Gambino R, Cassader M, et al. A novel approach to control hyperglycemia in type 2 diabetes: sodium glucose cotransport (SGLT) inhibitors: systematic review and meta-analysis of randomized trials. Ann Med 2012;44:375-93.

25. Invokana, INN-canagliflozin-WC500156456.pdf. http://www.ema. europa.eu/docs/en_GB/document_library/EPAR_-_Product Information/human/002649/WC500156456.pdf

26. Jardiance, INN-Empagliflozin_anx_128562_en.pdf. http://ec.europa eu/health/documents/community-register/2014/20140522128562/ anx_128562_en.pdf

27. Moher D, Liberati A, Tetzlaff J, et al. Preferred reporting items for systematic reviews and meta-analyses: the PRISMA statement. BMJ 2009;339:b2535.

28. ICTRP Search Portal. http://apps.who.int/trialsearch/

29. Wood L, Egger M, Gluud LL, et al. Empirical evidence of bias in treatment effect estimates in controlled trials with different interventions and outcomes: meta-epidemiological study. BMJ 2008;336:601-5.

30. The Nordic Cochrane Centre. Review Manager (RevMan). Copenhagen: 2011. 